\title{
Conflict resolved: On the role of spatial attention in reading and color naming tasks
}

\author{
Serje Robidoux ${ }^{1} \cdot$ Derek Besner $^{2}$
}

Published online: 11 April 2015

(C) Psychonomic Society, Inc. 2015

\begin{abstract}
The debate about whether or not visual word recognition requires spatial attention has been marked by a conflict: the results from different tasks yield different conclusions. Experiments in which the primary task is reading based show no evidence that unattended words are processed, whereas when the primary task is color identification, supposedly unattended words do affect processing. However, the color stimuli used to date does not appear to demand as much spatial attention as explicit word reading tasks. We first identify a color stimulus that requires as much spatial attention to identify as does a word. We then demonstrate that when spatial attention is appropriately captured, distractor words in unattended locations do not affect color identification. We conclude that there is no word identification without spatial attention.
\end{abstract}

Keywords Visual word recognition · Spatial attention · Color naming $\cdot$ Stroop interference $\cdot$ Exogenous cueing

The debate about whether spatial attention is necessary for stimulus identification extends for more than 60 years (Broadbent, 1954; Deutsch \& Deutsch, 1963). In visual word recognition, it has been marked by a conflict between the results of experiments using reading as the primary task and

Serje Robidoux

serje.robidoux@mq.edu.au

1 ARC Centre for Excellence in Cognition and its Disorders, Department of Cognitive Science, Macquarie University, Macquarie, NSW 2109, Australia

2 Department of Psychology, Cognition and Perception Unit (CPU), University of Waterloo, Waterloo, ON, Canada those involving color naming. When a subject has to identify a word that is spatially attended, researchers find no influence of a word appearing in a different location (Besner, Risko, \& Sklair, 2005; Lachter, Forster, \& Ruthruff, 2004; Lien, Ruthruff, Kouchi, \& Lachter, 2010; Waechter, Besner, \& Stolz, 2011). In contrast, when color naming is the task, there is consistent evidence that a word in a different location affects performance (Brown, Gore, \& Carr, 2002; Lachter, Ruthruff, Lien, \& McCann, 2008; Waechter et al., 2011). Many researchers take this latter result as evidence that identifying a word does not require spatial attention.

Waechter et al. (2011) suggested that the conflict between tasks arises from their relative attention demands, an idea that originated with Yantis and Johnston (1990). They proposed that color identification requires less spatial attention than word identification and that this allows subjects to distribute their spatial attention more broadly when color naming than when reading. This implies that the distractor words are not "unattended" when the task is color naming.

Robidoux, Rauwerda, and Besner (2014) reasoned that if Waechter et al. are correct, then a consequence is that the spatial cueing effect (the difference in reaction times between validly and invalidly cued trials) would be smaller when identifying colors than words. Using Posner's (1980) exogenous cueing paradigm, Robidoux and colleagues reported exactly that: color naming was always less sensitive to cue validity than was reading aloud. They concluded that color naming and word reading tasks had not been matched on the demand for spatial attention, challenging conclusions about "unattended" processing. Although unreported, we have tried several color patches in different contexts and found this pattern to be reliable - there is always an interaction between task and cueing such that color naming produces a smaller cueing effect than word identification (or nearly always as we will demonstrate). We take this 
to mean that the color naming tasks reported in the literature to date do not capture spatial attention as well as reading based tasks.

In this article, we first identify a target stimulus for color naming that requires as much spatial attention as does word reading. Following Waechter et al.'s (2011) hypothesis, we expect that this color patch will prevent processing of an unattended word. In a second experiment, we confirm that prediction. That is, when a color stimulus appropriately captures spatial attention, the results from color naming experiments become consistent with reading experiments: there is no evidence of word processing without attention.

\section{Defending the null: Bayes factors}

Our argument relies on establishing null effects: we first argue that our color patch produces a spatial cueing effect equivalent in size to the reading aloud task, implying $a$ null interaction between task and cue validity. In our second experiment, we demonstrate that there is no distractor effect from a word that is presented above or below a spatially attended location. Null effects are a challenge for frequentist statistical approaches. Fortunately, Bayesian statistical analysis offers a solution: one compares a null statistical model to a non-null model and determines which model is better supported by the data. A full description of Bayes factors and how they can be used to support null effects is beyond the scope of this article (see Morey \& Rouder, 2011, for a fuller treatment), but at a descriptive level, the Bayesian approach is to calculate the likelihood of observed data under the two assumptions separately and take their ratio. For example, in Experiment 1 we compare a model in which the interaction between cue validity and task is assumed to be zero (the null hypothesis model in conventional analyses) to a model that assumes the interaction between cue validity and task is non-zero (the usual alternative hypothesis). To the extent that the ratio of the likelihood of the null model to that of the alternative is greater than one $(>1)$, the data provide support for the null model, whereas values less than one $(<1)$ support the alternative hypothesis. Conveniently, Bayes factors have an intuitive interpretation: a Bayes factor of 7 implies that there is 7 times as much evidence for the null as for the alternative, whereas a Bayes factor of 0.5 suggests only half as much evidence for the null as the alternative (inversely, that there is twice as much evidence for the alternative). To obtain the Bayes factors for the present analyses, we used the $1 \mathrm{mBF}$ function from the BayesFactor package in R (Morey, Rouder, \& Jamil, 2014) with the default settings for all convergence and effect size parameters.

\section{Experiment 1}

The first step is to identify a color stimulus that requires as much spatial attention as word reading. In various experiments, we have tested different color stimulus presentations (colored \#\#\#\#\# strings, colored neutral words, and dualcolored patches à la Lachter et al., 2008) and task designs (intermixing vs. blocking the two tasks). Invariably, the results are a smaller cueing effect for color naming than for reading aloud (Robidoux et al., 2014), suggesting less demand for spatial attention. In this experiment, we introduce a color patch that demands as much spatial attention as word reading. Here, two colors are presented, as in Lachter et al. (2008); however, the arrangement of the colors is random. Participants are asked to name the dominant color (which makes up $67 \%$ of the display) and ignore the distractor color. The "target" screen in Fig. 1 provides an example of the stimulus. We hypothesized that uncertainty around the spatial location of the target color would require participants to focus their spatial attention more tightly in order to identify the dominant color. The results support this view.

\section{Method}

Participants Based on Robidoux et al. (2014), 32 undergraduate students from the University of Waterloo with normal or corrected-to-normal vision, normal color vision, and whose first language was English participated in the study for course credit. We note, however, that Bayesian analyses are not sensitive to stopping rule choices: data are collected until the results clearly favor one model over the other, or the experiment is abandoned as inconclusive (Dienes, 2014).

Design The experiment consisted of a 2 (Cue Validity: valid vs. invalid) x 2 (Task: reading aloud vs. color naming) withinparticipants factorial design, with reaction times (RT) and accuracy (\% error) as dependent measures. Task was blocked, and the order of the blocks counterbalanced across participants so that 16 participants named colors first, whilst the other 16 participants read words aloud first.

\section{Stimuli}

Word reading On each trial, participants were asked to read aloud one of four words ("pub," "close," "stop," "harder") in one of four colors (red, blue, green, or yellow). The four words were selected from the English Lexicon Project (Balota et al., 2007) to match the related color words on length, written frequency (from the Hypertext Analog Lexicon), and orthographic neighborhood size; and all items were within the typical range of reading aloud reaction times (RTs; 542-642 ms). Stimuli were displayed in Courier New, 14-point font. Target stimuli were approximately $1-\mathrm{cm}$ tall and ranged in width 


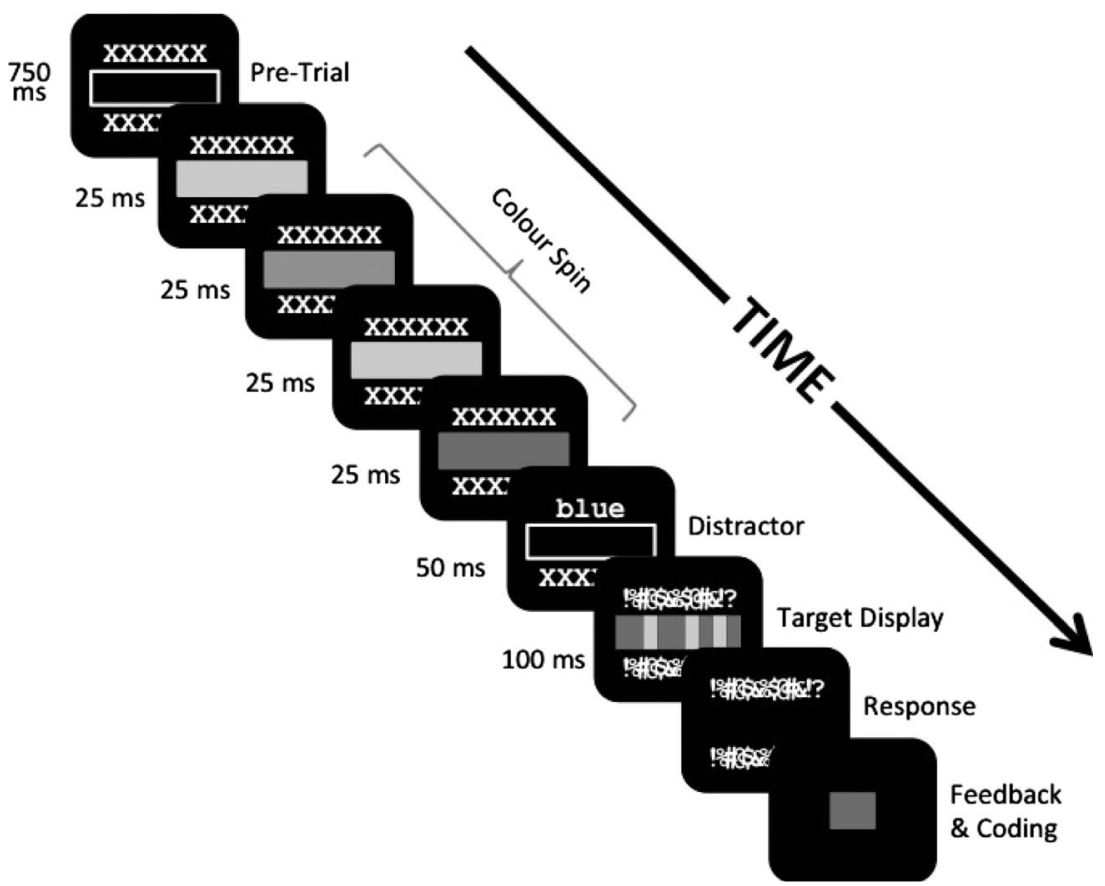

Fig. 1 Trial sequence used in Experiment 2. The grayscale center boxes appeared in color during the actual experiment. The empty white boxes appeared as depicted

from 2.5 to $5 \mathrm{~cm}$ ("harder"). Each word was presented in each color twice in each of the four conditions produced by crossing Cue Validity (valid vs. invalid) with Location (above vs. below fixation). This produced a total of 128 experimental trials.

Color naming Each trial consisted of a horizontal array of nine adjacent rectangles (without borders or gaps). The array was approximately $5-\mathrm{cm}$ wide, by $1-\mathrm{cm}$ high. For each trial, six of the rectangles were randomly assigned the target color (one of red, blue, green, or yellow). The remaining three rectangles were displayed in a single distractor color. Participants were instructed to name the color that made up the majority of the array. As with the four words, each color appeared as the target twice in each of the four Cue Validity by Location conditions. The distractor color was randomly selected from the three nontarget colors on each trial. This resulted in 128 experimental trials. In addition, each task block was preceded by a set of 16 practice trials that matched the presentation conditions of the experimental blocks, for a total of 288 trials per participant.

Procedure Participants were tested individually and seated at a comfortable distance from a computer monitor (approximately 50-60 cm). Each trial began with a fixation cross $(+)$ displayed at the center of the screen. The fixation cross remained on the screen for the duration of the trial. After $500 \mathrm{~ms}$, a light grey rectangle (approximately $3-\mathrm{cm}$ wide by $1-\mathrm{cm}$ high) flashed for $50 \mathrm{~ms}$ centered $3 \mathrm{~cm}$ either above or below the fixation cross; $50 \mathrm{~ms}$ after the removal of this cue, the target appeared in either the same location or in the opposite location and remained visible until response. The experimenter coded each response as correct, incorrect, or as a microphone misfire. This coding triggered the start of the next trial. Participants were instructed to keep their eyes on the fixation cross throughout each trial.

Participants completed the two tasks in separate blocks. Within each block, cue validity ( $50 \%$ valid) and target location (50\% top and bottom) were randomly intermixed. Each block began with a set of instructions describing the task for that block, followed by 16 practice trials. After the practice trials, the 128 experimental trials for that task were presented without a break, though participants could instruct the experimenter to withhold their coding if a break was desired.

Stimuli were displayed on a LCD monitor with a $60-\mathrm{Hz}$ refresh rate controlled by E-Prime 1.0 software (Psychology Software Tools, Pittsburgh, PA). Response times were collected to the nearest millisecond.

\section{Results}

Microphone errors resulted in the loss of $5.1 \%$ of trials. For the RT analysis, error trials were discarded $(1.6 \%$ of trials; summarized in Table 1). After removing errors and misfires, outliers were identified and removed, first by excluding RTs faster than $200 \mathrm{~ms}(0.17 \%$ of trials), and then by submitting the remaining RTs to the recursive technique recommended by Van Selst and Jolicoeur (1994), resulting in the elimination of an additional $1.0 \%$ of the trials. The remaining RTs (summarized in Table 1) were submitted to Bayesian analyses. 
Table 1 Mean reaction times (ms) and percentage errors (\%E) by task and cue validity for Experiment 1

\begin{tabular}{llllll}
\hline & \multicolumn{2}{l}{ Task } & & \\
\cline { 2 - 3 } & \multicolumn{2}{l}{ Reading aloud } & & & \multicolumn{2}{l}{ Color naming } \\
\cline { 2 - 3 } \cline { 5 - 6 } Cue validity & RT $(\mathrm{ms})$ & \%E & & RT $(\mathrm{ms})$ & $\% \mathrm{E}$ \\
\hline Invalid & 561 & 0.7 & & 691 & 1.9 \\
Valid & 526 & 0.9 & & 662 & 2.6 \\
Difference & 35 & -0.2 & & 29 & -0.7 \\
\hline
\end{tabular}

Specifically we compared two models, both with RTs as the dependent variable ${ }^{1}$, a main effect of the RT on the previous trial as a control variable, and the critical independent variables of Cue Validity and Task. In one model only the main effects were present, whereas the second model included the Cue Validity by Task interaction. In both models, Participant and Item intercepts were treated as random effects. Comparing the two models produced a Bayes Factor of 8.4, indicating very strong evidence for the null model. Due to very low error rates, accuracy was not analyzed.

\section{Discussion}

Previous research has interpreted distractor effects from "unattended" words in color-naming tasks as evidence that spatial attention is not needed to identify a word. However, Robidoux et al. (2014) demonstrated that the color stimuli used in these tasks do not require as much spatial attention as explicit word processing tasks where such distractor effects are not observed. Conversely, Experiment 1 demonstrates that by randomly varying the spatial arrangement of target and distractor colors in the target stimulus, we can match color naming to word reading on this dimension.

\section{Experiment 2}

Experiment 1 demonstrated that it is possible for color naming to require as much spatial attention as reading does. The critical question is whether or not this stimulus yields a word distractor effect, such as those found with the less attentionally demanding color patches. In Experiment 2, we followed the

\footnotetext{
${ }^{1}$ Though regression analyses in psycholinguistics are typically conducted using inverse or log transformed RTs, Balota, Aschenbrenner, and Yap (2013) have highlighted problems with this approach. Namely, these transformations compress interactions, sometimes reversing them from positive to negative. Here, when the inverse RT is used, the Bayes factor strongly favors a model with a reversed interaction so that the color naming task produces larger cueing effects than the word reading task. Regardless of which independent variable we adopt, the critical conclusion is the same: this new color patch requires at least as much spatial attention as our word reading task does.
}

procedures used by Lachter et al. (2008; Experiment 1), but replaced their color patch (a central color patch with an irrelevant border color), with the one used in Experiment 1. Evidence of a distractor effect in Experiment 2 would support the view that reading does not require spatial attention, because in Experiment 1 we demonstrated that the color patch commands spatial attention at least as much as do words. Conversely, the absence of distractor effects in Experiment 2 would support the view that reading requires spatial attention and that the previous demonstrations of unattended processing were the result of color stimuli that did not appropriately control spatial attention. Note that this logic once again relies on the presence of a null (no distractor effect). Consequently, we again rely on Bayes factors to analyze the results.

\section{Method}

Participants. Bayesian analysis eliminates concerns about stopping rules. We collected data until the analysis clearly favored one model over the other. This required 12 participants recruited through Macquarie University, with normal or corrected-to-normal vision, normal color vision, and whose first language was English. All participants were either awarded course credit or paid AUD $\$ 15$ for their participation.

Design The experiment consisted of a simple one factor (Distractor Type: congruent vs. incongruent) withinparticipants design, with RTs and accuracy as dependent variables.

\section{Stimuli}

Color patch: the color patch was identical to the one used in Experiment 1 except that we replaced the color green with pink due to green producing a disproportionate number of misfires in Experiment 1.

Distractor words: the distractor words were selected from the four color words red, yellow, blue, and pink.

Each color appeared as the target 48 times, paired with each possible distractor color 16 times, and each target-distractor color pairing appeared equally often on congruent and incongruent trials.

Table 2 Mean reaction times (ms) and percentage errors $(\% \mathrm{E})$ by distractor type for Experiment 2

\begin{tabular}{lll}
\hline Distractor type & RT $(\mathrm{ms})$ & $\% \mathrm{E}$ \\
\hline Incongruent & 579 & 2.9 \\
Congruent & 576 & 3.3 \\
Difference & 3 & -0.4 \\
\hline
\end{tabular}




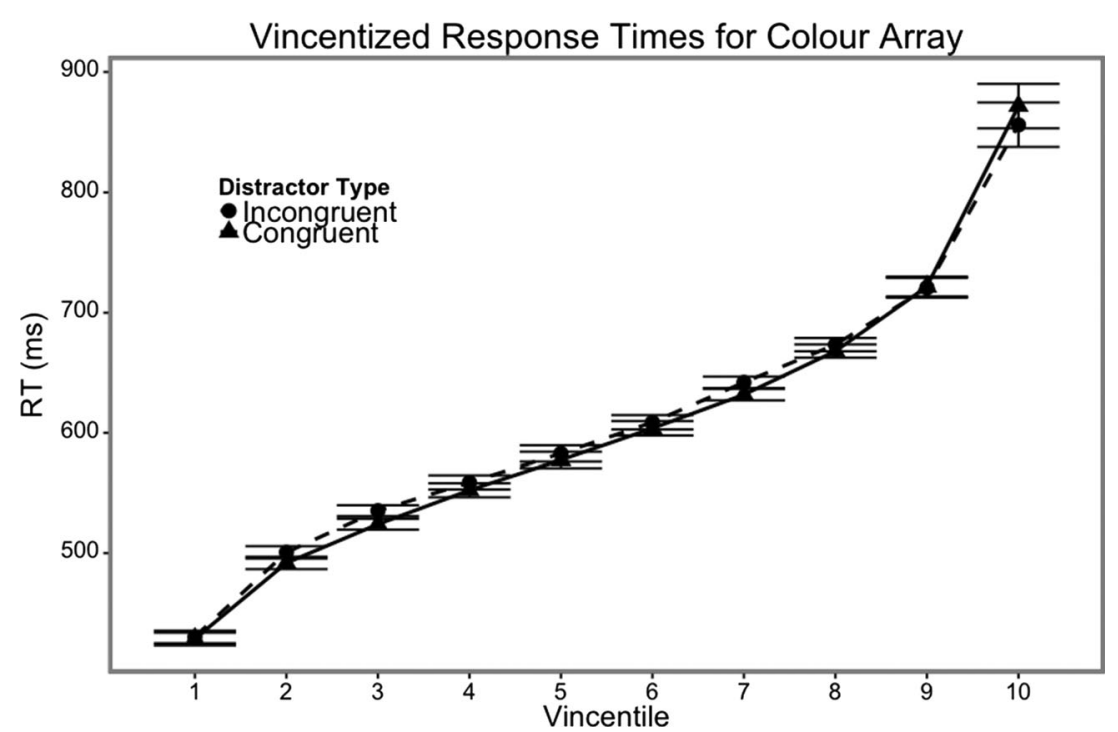

Fig. 2 Vincentized data from Experiment 2. The first vincentile represents the $10 \%$ fastest RTs for each subject, by distractor type (incongruent: dashed line with circles; congruent: solid lines and triangles); the second contains the next $10 \%$ fastest, and so on. Error

Procedure Participants were tested individually and were seated at a comfortable distance from a computer monitor (approximately 50-60 cm). The trial sequence was modeled on the one used by Lachter et al. (2008), and is depicted in Fig. 1. Trials began with a screen consisting of an unfilled white rectangle on a black background, and sequences of six X's appearing both above and below the rectangle. After $750 \mathrm{~ms}$, the color of the rectangle was randomly cycled through all four colors for $25 \mathrm{~ms}$ each. This was done to draw attention to the location of the target. Immediately after this $100 \mathrm{~ms}$ period, the white rectangle was presented again along with the distractor word in either the top or bottom location ( $50 \%$ of trials in each location). On half of the trials, the distractor word was congruent with the target color, on the other half the distractor word was one of the color words that was incongruent with both the target and the distractor color. That is, if the target stimulus was a mix of blue and yellow, then the incongruent distractor word could only be either "red" or "pink." After $50 \mathrm{~ms}$, both the top and bottom locations were replaced with overlapping strings (“\%@\&\$\#!” and “!\#\$\%@\&?”) acting as a backward mask. The target color patch appeared simultaneous with the onset of the backward mask and remained on the screen for $100 \mathrm{~ms}$ before being removed entirely. Participants were instructed to name the dominant color as quickly as possible (as in the color naming task in Experiment 1). After response, the target color appeared to allow the experimenter to code each response as correct, incorrect, or as a microphone misfire. The experimenter's coding triggered the start of the next trial. Participants were instructed to keep their eyes on the rectangle in the center of the screen throughout each trial. bars represent the standard error of the differences within each vincentile, although these should be interpreted with caution in light of intercorrelations between the vincentile bins

The experiment began with a set of instructions describing the task, followed by 12 practice trials to familiarize the participants with the trial sequence. After the practice trials, the 192 experimental trials were presented with three breaks.

Stimuli were displayed on a LCD monitor with a $60-\mathrm{Hz}$ refresh rate controlled by E-Prime 1.0 software (Psychology Software Tools, Pittsburgh, PA). Response times were collected to the nearest millisecond.

\section{Results}

Data preparation followed the same procedures as in Experiment 1. Microphone misfires (2.4\%) and errors (3.1\%) were discarded along with RTs faster than $200 \mathrm{~ms}$ ( $0.7 \%$ of trials). For the RT analysis, the remaining trials were submitted to the recursive outlier removal technique recommended by Van Selst and Jolicoeur (1994), eliminating an additional $1.6 \%$ of the trials. The remaining RTs (summarized in Table 2) were submitted to Bayesian analysis. We

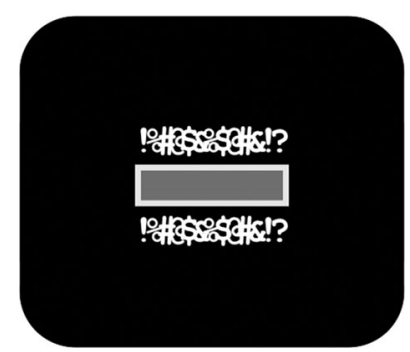

Fig. 3 Depiction of the target display used for Experiment 3. The participant identified the color of the internal rectangle while ignoring the external border. The target color makes up $66 \%$ of the color patch 
Table 3 Mean reaction times (ms) and percentage errors (\%E) by distractor type for Experiment 3. Due to the very low error rates, accuracy was not analyzed

\begin{tabular}{lll}
\hline Distractor type & RT $(\mathrm{ms})$ & $\% \mathrm{E}$ \\
\hline Incongruent & 580 & 3.6 \\
Congruent & 559 & 4.4 \\
Difference & 21 & -0.8 \\
\hline
\end{tabular}

compared two models: one in which RT was modeled with a main effect of the previous trial's RT as a control variable and the critical Distractor Type manipulation. In the second model, only the effect of previous RT was included. In both models, Participant and Item intercepts were treated as random effects. Comparing the two models produced a Bayes factor of 16.8, indicating very strong evidence against the distractor effect. Due to low error rates, accuracy was not analyzed.

\section{RT distribution}

It is possible that the present results are due to different effects throughout the reaction time distribution. That is, the effect may be present in some parts of the distribution but depressed or even reversed in other parts of the distribution (see Yap, Balota, Tse \& Besner, 2008 for a demonstration of such a pattern). To assess this possibility, we sorted the response times by condition and subject and grouped them into ten roughly equal-sized bins (so-called Vincentizing). Figure 2 depicts the results across the distribution of RTs. The present results are clearly not due to a more complex pattern in the distribution of reaction times.

\section{Discussion}

Based on Experiments 1 and 2, it is clear that when a color patch that appropriately captures attention is used, distractor effects are eliminated. Furthermore, this is not simply a shrinking of the effect so that it is more difficult to detect; there is no congruency effect anywhere across the distribution of RTs.

We have taken pains to match our paradigm to Lachter et al.'s (2008) reported Experiment 1 in all critical respects, but it is possible that some feature of our apparatus or methodology has eliminated the distractor effect in a way that has nothing to do with the color patch design. In Experiment 3, we return to Lachter et al.'s color patch (2008) to ensure that we are able to replicate their result.

\section{Experiment 3}

\section{Method}

Participants. As in Experiment 2, we collected data until the planned Bayesian analyses clearly favored one model over the other. The participants consisted of 14 subjects recruited through Macquarie University, with normal or corrected-tonormal vision, normal color vision, and whose first language was English. Participants were awarded course credit or paid AUD $\$ 15$ for their participation.

Design The experiment consisted of a one factor (Distractor Type: congruent vs. incongruent) within-participants design, with RTs and accuracy as dependent variables.

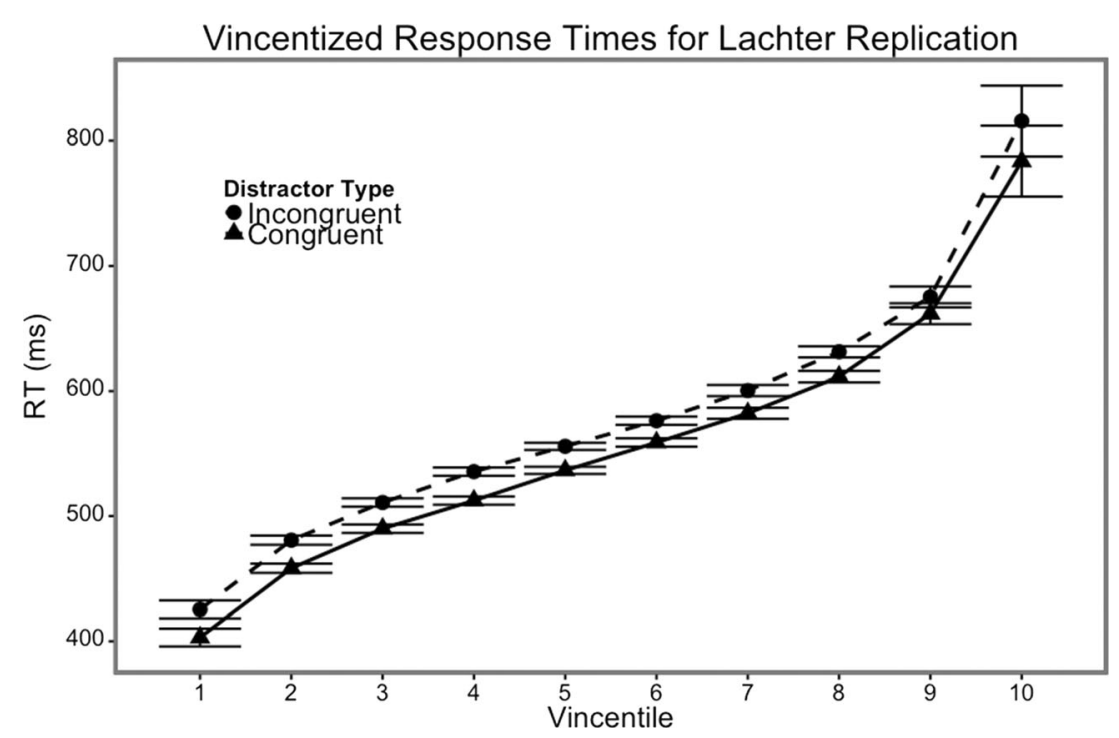

Fig. 4 Vincentized data from Experiment 3. The first vincentile represents the $10 \%$ fastest RTs for each subject, by distractor type (incongruent: dashed line with circles; congruent: solid lines and triangles); the second contains the next $10 \%$ fastest, and so on. Error bars represent the standard error of the differences within each vincentile, although these should be interpreted with caution in light of intercorrelations between the vincentile bins 
Stimuli The stimuli were identical to those in Experiment 2 except for the arrangement of the target and distractor colors in the target color patch. Rather than the random array arrangement, the patch consisted of a centrally presented rectangle in the target color $(4.8 \mathrm{~cm}$ by $1.0 \mathrm{~cm})$, surrounded by a border $(0.2 \mathrm{~cm})$ in the distractor color (Fig. 3). This patch is identical to the patch used in Robidoux et al. (2014), Experiment 2.

Procedure Procedures were identical to those described in Experiment 2.

\section{Results}

As before, we eliminated microphone misfires $(0.8 \%)$ and errors $(4.0 \%)$ along with RTs faster than $200 \mathrm{~ms}(0$ in this case). For the RT analysis, the remaining trials were submitted to the Van Selst and Jolicoeur (1994) outlier procedure, resulting in the elimination of an additional $1.5 \%$ of the trials. The remaining RTs (summarized in Table 3 ) were analyzed as in Experiment 2. We compared two models: one in which RT was modeled with a main effect of the previous trial's RT as a control variable, and the critical Distractor Type manipulation. In the second model, only the effect of previous RT was included. In both models, Participant and Item intercepts were treated as random effects. Here, the Bayes Factor was 0.000026 , implying more than 38,000 times as much evidence for the presence of a congruence effect over the null model. Due to the low error rates, accuracy was not analyzed.

\section{RT distribution}

As in Experiment 2, we again examined the distribution of RTs to determine whether there were any unusual characteristics. Figure 4 depicts the reaction times as a function of vincentiles. It is clear that the congruency effect is not the result of any unusual distributional characteristics.

\section{General discussion and conclusion}

During the past decade, there has been a divide in the literature about whether or not word identification requires spatial attention. Experiments in which subjects were required to identify a target word in the presence of unattended distractor words produce no evidence of unattended processing when spatial attention is firmly on the target (Besner et al., 2005; Lachter et al. 2004, Lien et al., 2010; Waechter et al. 2011). On the other hand, when color naming is the task, distractor words consistently interfere with processing (Brown, Gore, \& Carr, 2002; Lachter et al., 2008; Waechter et al., 2011, Experiment $5)$. However, following Waechter et al.'s hypothesis, Robidoux et al. (2014) showed that spatial cueing effects are smaller for color identification than for word identification.
They concluded that a simple color identification task may not appropriately control spatial attention - that is, putatively "unattended" stimuli may be attended. We extend that line of argument.

In Experiment 1, we introduced a new color stimulus and demonstrated that it requires as much spatial attention to process as do words when reading aloud (indexed by the Posner spatial cueing paradigm). In Experiment 2, we demonstrated that the new color patch shows no evidence of distractor word processing. Using Bayesian statistical methods, we are confident that this represents a true null. Finally, in Experiment 3 we replicate the original Lachter et al. (2008) result, clearly demonstrating that it is the nature of the color patch that is the critical difference. Taken together, the present experiments allow only one conclusion: there is no word processing without spatial attention.

Acknowledgments The authors thank Robert McCann and two anonymous reviewers for their helpful comments on an earlier version of this article. Funding for this project was provided by the Australian Research Council Centre of Excellence in Cognition and its Disorders [CE110001021], and Natural Sciences and Engineering Research Council of Canada [AO998].

\section{References}

Balota, D. A., Aschenbrenner, A. J., \& Yap, M. J. (2013). Additive effects of word frequency and stimulus quality: The influence of trial history and data transformations. Journal of Experimental Psychology: Learning, Memory, and Cognition, 39(5), 1563-1571. doi:10.1037/ a0032186

Balota, D. A., Yap, M. J., Cortese, M. J., Hutchison, K. A., Kessler, B., Loftis, B., \& Treiman, R. (2007). The English Lexicon Project. Behavior Research Methods, 39(3), 445-459.

Besner, D., Risko, E. F., \& Sklair, N. (2005). Spatial attention as a necessary preliminary to early processes in reading. Canadian Journal of Experimental Psychology/Revue canadienne de psychologie expérimentale, 59(2), 99-108. doi:10.1037/h0087465

Broadbent, D. E. (1954). The role of auditory localization in attention and memory span. Journal of Experimental Psychology, 47(3), 191196.

Brown, T. L., Gore, C. L., \& Carr, T. H. (2002). Visual attention and word recognition in Stroop color naming: Is word recognition "automatic?". Journal of Experimental Psychology: General, 131(2), 220240. doi:10.1037/0096-3445.131.2.220

Deutsch, J. A., \& Deutsch, D. (1963). Attention: Some theoretical considerations. Psychological Review, 70(1), 80-90.

Dienes, Z. (2014). Using Bayes to get the most out of non-significant results. Frontiers in Psychology, 5, 781. doi:10.3389/fpsyg.2014. 00781

Lachter, J., Forster, K. I., \& Ruthruff, E. (2004). Forty-five years after Broadbent (1958): Still no identification without attention. Psychological Review, 111(4), 880-913. doi:10.1037/0033-295X. 111.4.880

Lachter, J., Ruthruff, E., Lien, M., \& McCann, R. S. (2008). Is attention needed for word identification? Evidence from the Stroop paradigm. Psychonomic Bulletin \& Review, 15(5), 950-955. doi:10.3758/PBR. 15.5 .950 
Lien, M., Ruthruff, E., Kouchi, S., \& Lachter, J. (2010). Even frequent and expected words are not identified without spatial attention. Attention, Perception, \& Psychophysics, 72(4), 973-988.

Morey, R. D., \& Rouder, J. N. (2011). Bayes factor approaches for testing interval null hypotheses. Psychological Methods, 16(4), 406-419. doi: $10.1037 / \mathrm{a} 0024377$

Morey, R. D., Rouder, J. N., \& Jamil, T. (2014). BayesFactor: Computation of Bayes factors for common designs. R package version 0.9 .8

Posner, M. I. (1980). Orienting of attention. The Quarterly Journal of Experimental Psychology, 32(1), 3-25.

Robidoux, S., Rauwerda, D., \& Besner, D. (2014). Basic processes in reading aloud and colour naming: Towards a better understanding of the role of spatial attention. The Quarterly Journal of Experimental Psychology, 67(5), 979-990. doi:10.1080/17470218.2013.838686
Van Selst, M., \& Jolicoeur, P. (1994). A solution to the effect of sample size on outlier elimination. The Quarterly Journal of Experimental Psychology A: Human Experimental Psychology, 47(3), 631-650.

Waechter, S., Besner, D., \& Stolz, J. A. (2011). Basic processes in reading: Spatial attention as a necessary preliminary to orthographic and semantic processing. Visual Cognition, 19(2), 171-202. doi:10. 1080/13506285.2010.517228

Yantis, S., \& Johnston, J. C. (1990). On the locus of visual selection: Evidence from focused attention tasks. Journal of Experimental Psychology: Human Perception and Performance, 16(1), 135149. doi:10.1037/0096-1523.16.1.135

Yap, M. J., Balota, D. A., Tse, C., \& Besner, D. (2008). On the additive effects of stimulus quality and word frequency in lexical decision: Evidence for opposing interactive influences revealed by RT distributional analyses. Journal of Experimental Psychology: Learning, Memory, and Cognition, 34(3), 495-513. doi:10.1037/0278-7393.34.3.495 